\title{
A Vasoactive Intestinal Peptide-like Cotransmitter at Cholinergic Synapses Between Rat Myenteric Neurons in Cell Culture
}

\author{
Alan L. Willard \\ Department of Physiology, University of North Carolina, Chapel Hill, North Carolina 27599
}

\begin{abstract}
Intracellular recording and immunochemical techniques were used to study synaptic transmission between individual pairs of rat myenteric plexus neurons in cell culture. This report describes the synaptic connections made by "dual function" presynaptic neurons that evoked slow postsynaptic depolarizations (slow EPSPs) in the same neurons in which they also evoked fast nicotinic cholinergic EPSPs. The slow EPSPs occurred only when presynaptic neurons were stimulated at frequencies of $5 \mathrm{~Hz}$ or higher. During the slow EPSPs, slope input resistance increased. The slow EPSPs were not detectably voltage-dependent, and they reversed sign at the estimated $\mathrm{K}^{+}$equilibrium potential, suggesting that they resulted from a synaptically mediated decrease in resting $\mathbf{K}^{+}$ conductance.

Several lines of evidence suggested that dual-function neurons evoke slow EPSPs by releasing a vasoactive intestinal peptide (VIP)-like cotransmitter. (1) Immunocytochemical staining revealed VIP-like immunoreactivity in all physiologically identified dual-function neurons. (2) Responses to exogenously applied VIP mimicked the slow EPSPs. (3) Superfusion of cultures with anti-VIP antisera blocked the slow EPSPs reversibly, as did application of desensitizing doses of VIP. These findings suggest that during periods of increased activity, subsets of cholinergic myenteric neurons release a VIP-like cotransmitter that enhances postsynaptic excitability. The effects of the cotransmitter may help to compensate for decreases in nicotinic EPSPs that occur during increased presynaptic activity.
\end{abstract}

In addition to "classical" neurotransmitters such as ACh, GABA or norepinephrine, many individual neurons contain one or more pharmacologically active peptides (reviewed by Hökfelt et al., 1987). Although most colocalized peptides have unknown functions, it is often hypothesized that neurons release peptides and classical transmitters as "cotransmitters." Attempts to demonstrate peptidergic cotransmission have met with mixed success. In several systems, including neuromuscular junctions of cockroach (Adams and O'Shea, 1983) and leech (Li and Calabrese, 1987), lumbar sympathetic ganglia of bullfrog (Jan et

\footnotetext{
Received July 10, 1989; revised Sept. 27, 1989; accepted Sept. 28, 1989.

This work was supported by NIH grant NS24362 and an Alfred P. Sloan Fellowship in Neuroscience. I thank Ms. Laura Fleck for technical assistance, Dr. Larry Marshall and students in my laboratory for critical comments on an earlier draft of the manuscript, and Drs. P. Hogan and M. Epstein for generous donations of VIP antisera.

Correspondence should be addressed to Dr. Alan L. Willard, Department of Physiology, CB \#7545, University of North Carolina, Chapel Hill, NC 275997545

Copyright (C) 1990 Society for Neuroscience $0270-6474 / 90 / 031025-10 \$ 02.00 / 0$
}

al., 1979, 1983; Jan and Jan, 1982) and salivary glands of cat (Lundberg et al., 1980), neurons release colocalized peptides to evoke postsynaptic responses distinct from those caused by their classical cotransmitters. However, Bowers et al. (1986) showed that release of a colocalized substance P-like peptide from cholinergic terminals in frog cardiac ganglia docs not cause an electrophysiologically detectable postsynaptic response in neurons innervated by those terminals. They concluded that either the peptide acts upon non-neuronal targets or it causes electrically silent postsynaptic responses. Clearly, more studies of the synaptic actions of neurons containing multiple transmitter candidates will be required before we can know the full range of physiological functions of colocalized peptides.

Important questions about the physiology of colocalized peptides include the following: Do the same stimuli cause release of both peptides and classical transmitters? Do cotransmitters act upon identical targets? Do they have complementary or antagonistic actions? Can peptides and classical cotransmitters regulate each other's release? Such questions are only beginning to be addressed, chiefly because demonstrating peptidergic $\mathrm{co}$ transmission is more difficult than demonstrating peptidergic transmission. For example, stimulation of mixed nerve bundles evokes both fast cholinergic and slow noncholinergic synaptic potentials in a variety of autonomic neurons (e.g., Nishi and Koketsu, 1968; Neild, 1978; Johnson et al., 1980; Mihara et al., 1985; Dreyer and Chiappinelli, 1985). Peptides probably mediate some of the slow postsynaptic responses evoked by such stimuli, but in most cases it is not known whether those peptides are relcased by themsclves or with classical cotransmitters (e.g., Bornstein et al., 1984; Love and Szurszewski, 1987). Few intact neural systems lend themselves to rigorous demonstrations of cotransmission because one must be able both to identify preand postsynaptic cells reliably and to stimulate selectively only those presynaptic neuron(s) containing the hypothesized cotransmitters. The most useful preparations have been invertebrate nervous systems that contain reliably identifiable individual neurons (e.g., Adams and O'Shea, 1983; Bishop et al., 1987; $\mathrm{Li}$ and Calabrese, 1987) and those portions of the vertebrate peripheral nervous system that contain nearly homogeneous populations of presynaptic axons, such as visceromotor or autonomic preganglionic nerves (Jan et al., 1979, 1983; Lundberg et al., 1980; Jan and Jan, 1982). However, although there are several neuronal systems in which individual presynaptic axons elicit both fast and slow EPSPs (e.g., Dreyer and Chiapinelli, 1985; Brookes et al., 1988; Nusbaum and Marder, 1989), to date there is only one system, the bullfrog lumbar sympathetic ganglion, in which it has been possible to identify rigorously both a fast classical and a slow peptidergic cotransmitter at 
synaptic connections between neurons (Jan et al., 1979, 1983; Jan and Jan, 1982).

This study used cell cultures to investigate cotransmission between myenteric neurons. Neurons in cell cultures are readily accessible to electrophysiological, pharmacological, and immunological probes. This accessibility permits studies of synaptic interactions between individual neurons to be followed by immunocytochemical testing of physiologically identified presynaptic neurons for content of suspected transmitters or cotransmitters. Cell cultures have been useful for analysis of fast synaptic interactions between individual nerve cells (e.g., O'Lague et al., 1978; Nelson et al., 1983, 1986; Willard and Nishi, 1985b; Hirano et al., 1986; O'Brien and Fischbach, 1986) and for analysis of slow synaptic interactions between sympathetic neurons and cardiac myocytes (Matsumoto et al., 1987; Sah and Matsumoto, 1987). However, slow synaptic interactions between neurons in cell culture have not been studied in detail. To date, myenteric neurons are the only neurons reported to cause slow synaptic potentials in other neurons in culture. During studies of synaptic interactions between cultured myenteric neurons, Willard and Nishi (1985b, 1987) observed "dual function" neurons that evoked fast nicotinic EPSPs and, when stimulated at higher frequencies, slow noncholinergic EPSPs. The slow EPSPs resembled the responses evoked by application of several neurotransmitter candidates found in the cultures, including substance P, VIP, and serotonin (Willard and Nishi, 1985b). However, only one of those substances, VIP, was detectable by immunocytochemical examination of physiologically identified dual-function neurons (Willard and Nishi, 1987).

The purpose of the present study was to examine the synaptic connections of dual-function myenteric neurons and to test the hypothesis that they use a VIP-like molecule as a cotransmitter. The main conclusions are that (1) different patterns of presynaptic stimuli are optimal for evoking release of $\mathrm{ACh}$ or of a noncholinergic cotransmitter from dual-function myenteric neurons, (2) the noncholinergic cotransmitter causes slow depolarizations by decreasing a resting $\mathrm{K}^{+}$conductance, and (3) the cotransmitter is a VIP-like molecule.

\section{Materials and Methods}

Cell cultures. Tissue strips containing smooth muscle and myenteric plexus were removed from the small intestines of 1- to 3-d-old rat pups (CD strain; Charles River) as described by Nishi and Willard (1985). Suspensions of single cells were obtained by the following shortened version of their protocol. (1) Tissue strips were incubated $20 \mathrm{~min}$ at $37^{\circ} \mathrm{C}$ in $\mathrm{Ca}, \mathrm{Mg}$-free Hanks' Balanced Salt Solution containing $4 \mathrm{mg} / \mathrm{ml}$ of Dispase (Bochringer-Mannheim) and then triturated gently 10 times. (2) The Dispase solution was replaced and the incubation was continued for an additional 60 or 90 min with manual agitation of the tubes at $10 \mathrm{~min}$ intervals and trituration $(30 \times)$ at $30 \mathrm{~min}$ intervals. If the strips were judged to be inadequately dissociated after the trituration at 60 $\mathrm{min}$, they were incubated for another $30 \mathrm{~min}$. More than $90 \mathrm{~min}$ of incubation in Dispase was never necessary. (3) Cells were removed from the Dispase by centrifugation, and the resulting pellet was then gently resuspended in $3.5 \mathrm{ml}$ of plating medium and filtered through 2 circles of 100 and $33 \mu \mathrm{m}$ Nitex mesh (Tetko) in Swinnex filter holders.

Cells were counted in a hemocytometer, and the density of the cell suspension was adjusted to 300,000 cells $/ \mathrm{ml}$. Cells $(30,000$ in $0.1 \mathrm{ml})$ were then placed inside rings of paraffin $(7 \mathrm{~mm}$ i.d.; $10 \mathrm{~mm}$ o.d.) that confined them to the centers of $35 \mathrm{~mm}$ culture dishes. The paraffin rings contained a film of rat tail collagen prepared as previously described (Nishi and Willard, 1985). Each paraffin ring was surrounded by a 16 $\mathrm{mm}$ glass ring. The glass ring, which allowed medium to seep slowly under it, helped minimize the number of cells flushed out of the paraffin ring when medium was added to the rest of the dish.
Three days after plating, when the neurons were well attached to the substratum, the glass rings were removed, and the plating medium, which contained mitotic inhibitors, was replaced with growth medium, which lacked mitotic inhibitors (Nishi and Willard, 1985). Culture medium was replaced twicc wcckly thereafter.

Electrophysiology. Experiments were done at room temperature on the stage of an inverted microscope (Nikon Diaphot) that had epifluorescence and Hoffman modulation contrast optics. To facilitate placement of electrodes, the upper $5 \mathrm{~mm}$ of the sides of culture dishes were cut away with a hot needle. Culture medium was replaced with recording solution (see below).

Patch electrodes were made from hematocrit glass (Fisher Blue Tip) and had DC resistances of 4-8 M 2 when filled with internal solution. Recordings were made in the "whole cell" configuration (Hamill et al., 1981). Patch electrodes were also used for extracellular stimulation of neurons as described by Role and Fischbach (1987). Current-clamp recordings were made either with a Dagan 8900 patch-clamp amplifier with a head stage containing a $100 \mathrm{M} \Omega$ feedback resistor or with a WPI M-707A electrometer.

Identification of synaptically connected pairs of neurons. Synaptically connected pairs of neurons were located either by testing randomly selected pairs of neurons for interactions or by the following procedure. Patch pipets containing $0.12 \%$ Lucifer yellow (LY) in normal internal solution were used to inject LY into a neuronal cell body for $2 \mathrm{~min}$ or less. (Cells not brightly stained within 2 min were abandoned because they inevitably showed signs of damage such as loss of resting potential and diminished action potentials.) Dye was allowed to diffuse into the processes and terminals of injected cells for about $15 \mathrm{~min}$. The field of view was then examined briefly $(<10 \mathrm{sec})$ to determine whether any neuronal cell bodies were contacted by varicosities of the injected cell. Intracellular recordings were then made from the contacted cell while the injected cell was stimulated either with an intracellular electrode or with a loosely sealed ( $<200 \mathrm{M} \Omega$ ) cxtraccllular pipet as described by Role and Fischbach (1987). Pipets used for stimulation were connected to an electrometer to permit monitoring of action potentials in the stimulated cell.

Three criteria determined whether fast EPSPs were judged to be monosynaptic: whether they occurred at brief $(<3 \mathrm{msec})$ fixed latencies, whether they could be evoked reliably at frequencies above $5 \mathrm{~Hz}$, and whether they persisted in concentrations of hexamethonium that reduced their amplitudes by $50-80 \%$ (see Willard and Nishi, 1987). All fast EPSPs in these cultures were cholinergic: ACh responses mimicked them, 0.5-10 $\mu \mathrm{M}$ hexamethonium reduced their amplitudes, and 20-50 $\mu \mathrm{M}$ hexamethonium completely blocked them (Willard and Nishi, 1985b, 1987). Noncholinergic fast EPSPs were never observed.

Injected cells survived well and the synaptic connections made by them were as robust as those made by uninjected cclls provided that the following precautions were taken: (1) limiting injection times to 2 min or less; (2) limiting exposure to fluorescent illumination to less than $10 \mathrm{sec}$; (3) working in a darkened room and being fully dark adapted before inspecting the injected cell; and (4) using the microscope illuminator (at the lowest useful intensity) only while positioning electrodes and microperfusion pipets. When varicosities of an injected cell contacted the cell body of another neuron, the axon connecting the varicosities usually appeared to "bend" around the contacted cell body. With experience, it was possible to recognize such structures almost instantly. The neurons were then viewed with Hoffman optics to confirm that there was indeed a cell body at the site of the "bend."

Application of test solutions. Fresh recording solution or test solutions were added to the entire culture dish via a gravity-driven perfusion system. Often, it was desirable to restrict test solutions to the arca of the dish inside the paraffin ring. This was accomplished by removing all fluid from the dish, except for about $30 \mu \mathrm{l}$ in the paraffin ring, and then flooding the ring via a microperfusion system consisting of 3 lines of PE 10 polyethylene tubing epoxied into a glass tip (100 $\mu \mathrm{m}$ i.d.). The dead space in the tip was less than $3 \mu \mathrm{l}$. In other experiments, test solutions were pressure ejected from micropipets connected to a PicoSpritzer.

The recording solution contained $137 \mathrm{~mm} \mathrm{NaCl}, 5.4 \mathrm{~mm} \mathrm{KCl}, 0.44$ mM KH${ }_{2} \mathrm{PO}_{4}, 0.34 \mathrm{~mm} \mathrm{Na}_{2} \mathrm{HPO}_{4}, 10 \mathrm{~mm}$ glucose, $2.5 \mathrm{~mm} \mathrm{CaCl}_{2}, 5 \mathrm{~mm}$ HEPES-NaHEPES (pH 7.35), and $28 \mu \mathrm{M}$ phenol red. Test solutions of peptides also contained $1-2.5 \mathrm{mg} / \mathrm{ml}$ of BSA.

The internal solution contained $150 \mathrm{~mm} \mathrm{KCl}, 1 \mathrm{mM}$ EGTA-KOH, 2 $\mathrm{mm} \mathrm{MgCl}_{2}, 2 \mathrm{~mm} \mathrm{Na} \mathrm{ATP}_{2}$, $5 \mathrm{~mm}$ HEPES (pH 7.35), and $28 \mu \mathrm{M}$ phenol red. 
Immunocytochemistry. Methods for immunocytochemical staining and relocation of electrophysiologically characterized cells were described previously (Nishi and Willard, 1985; Willard and Nishi, 1987). In some experiments, binding of primary antisera was visualized with an avidin-biotin $(\mathrm{ABC})$ peroxidase procedure rather than with a peroxidase-antiperoxidase procedure. In the $\mathrm{ABC}$ experiments, the staining was performed according to the protocol provided with the $A B C$ kit by Vector Laboratories.

Staining was considered specific only if it was blocked by preadsorbing the primary antiserum with the antigen against which it had been raised and not by preadsorption with other similar molecules. Preincubation with synthetic VIP $(1 \mu \mathrm{g} / \mathrm{ml})$ abolished immunocytochemical staining with all 3 anti-VIP antisera used. Preincubation with $1 \mu \mathrm{g} / \mathrm{ml}$ of secretin or peptide histidine-isoleucine (PHI) did not detectably affect staining. Other controls included omitting the primary antiserum in order to test for nonspecific binding of the secondary antiserum.

Materials. Peptides were purchased from Peninsula Laboratories and from Sigma. Three anti-VIP antisera were used. Antiserum $H$ was raised against a carbodiimide conjugate of synthetic VIP and BSA by Dr. P. Hogan of Harvard Medical School. Antiserum M was raised against a carbodiimide conjugate of synthetic VIP to thyroglobulin by Drs. J. Dahl and M. Epstein of the University of Wisconsin. Antiserum S was purchased from Sero-tec and was raised against a glutaraldehyde conjugate of authentic porcine VIP. Anti-substance $P$ antisera were purchased from IncStar and Peninsula. Normal rabbit serum was purchased from Dako and Vector. All other chemicals were purchased from Sigma.

\section{Results}

This report presents results obtained from 42 pairs of neurons in cultures from 7 different platings. All the presynaptic neurons in this study were "dual function": they evoked both nicotinic fast EPSPs and noncholinergic slow depolarizations in postsynaptic neurons. In contrast, "monofunctional" cholinergic neurons evoked only fast EPSPs. The ease of finding dual-function neurons varied considerably among cultures from different platings. In cultures from 19 other platings, none of 97 cholinergic neurons tested were dual function. The reason for such platingto-plating variability in the incidence of dual-function neurons is unknown.

\section{Properties of slow depolarizations (slow EPSPS) evoked by dual-function neurons}

Dual-function neurons werc identificd by thcir ability to evoke both fast nicotinic EPSPs and slow postsynaptic depolarizations. During slow depolarizations evoked by dual-function neurons, postsynaptic excitability increased. Previously subthreshold current pulses or nicotinic EPSPs often became suprathreshold, postsynaptic neurons fired more action potentials during suprathreshold current pulses, and some postsynaptic neurons began to fire action potentials spontaneously. Thus, the slow depolarizations are called slow EPSPs because of their excitatory effects on postsynaptic neurons.

Frequency dependence of cotransmission. Figure 1 shows that increasing the frequency of stimulation of dual-function neurons had opposite effects on the amplitudes of cholinergic and noncholinergic postsynaptic responses. Amplitudes of the monosynaptic fast EPSPs declined during stimulation of presynaptic neurons at frequencies above $0.5 \mathrm{~Hz}$. Similar frequency-dependent declines in the amplitudes of fast EPSPs also occur in the myenteric plexus of guinea pigs (Nishi and North, 1973; Hanani and Burnstock, 1985) and rats (Brookes et al., 1988). Nishi and North (1973) suggested that the decrease results from decreased release of $\mathrm{ACh}$ rather than from decreased postsynaptic sensitivity to $\mathrm{ACh}$ because responses of postsynaptic neurons to ionophoretic application of $\mathrm{ACh}$ did not decrease.

In contrast to fast EPSPs, slow EPSPs increased in amplitude

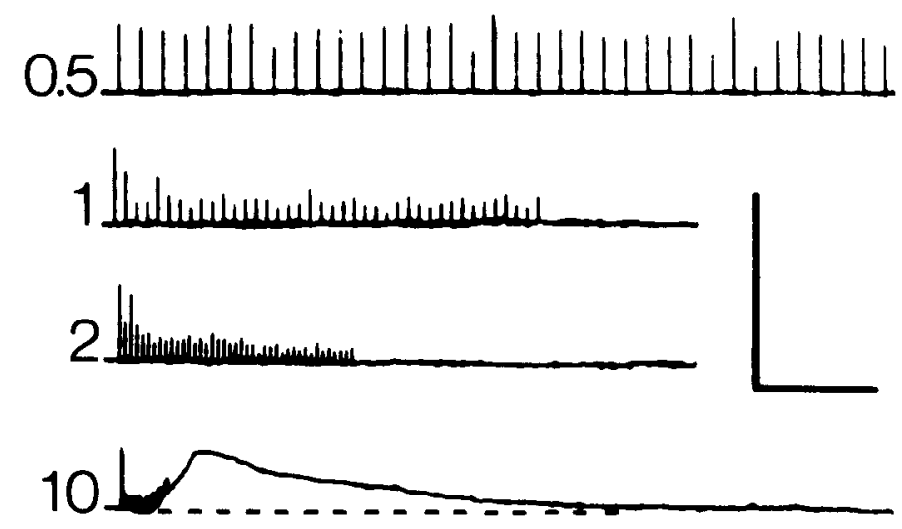

Figure 1. Frequency dependence of slow EPSPs. The traces are chart records of responses of a postsynaptic neuron to trains of 40 action potentials elicited by extracellular stimulation of a dual-function presynaptic neuron. The frequency of action potentials within the trains, indicated to the left of each trace, was varied from 0.5 to $10 \mathrm{~Hz}$. Presynaptic action potentials reliably evoked monosynaptic fast EPSPs, which appear as rapid upward deflections, at all frequencies of stimulation. However, the amplitudes of fast EPSPs declined at stimulation frequencies greater than $0.5 \mathrm{~Hz}$. At $10 \mathrm{~Hz}$, the train also evoked a slow EPSP. Calibration bars: $20 \mathrm{mV}, 10 \mathrm{sec}$.

when the frequency of presynaptic stimulation was increased. Rundown (see Fig. 2 and section below) prevented extensive analysis of the relative contributions of stimulus frequency and duration to the amplitude and duration of slow EPSPs, but the following 2 generalizations can be made: (1) Increasing stimulus frequency reduced the minimum number of action potentials required to elicit detectable slow EPSPs and increased the amplitudes and durations of slow EPSPs evoked by a given suboptimal number of action potentials, and (2) increasing the duration of stimulation at a fixed frequency had larger effects on durations than on amplitudes of slow EPSPs.

The preceding generalizations were based upon results obtained from 28 dual-function neurons stimulated at 1, 2, 5, 10 , and $20 \mathrm{~Hz}$ for up to $60 \mathrm{sec}$. None evoked slow EPSPs when stimulated at 1 or $2 \mathrm{~Hz}$. When stimulated at $5 \mathrm{~Hz}, 6$ neurons evoked detectable ( $\geq 2 \mathrm{mV}$ ) slow EPSPs. For those 6 neurons, the mean minimum number of action potentials (APs) required to evoke slow EPSPs at 5,10 , and $20 \mathrm{~Hz}$ was $204 \pm 64$ (SD; range, 125-300), $56 \pm 27$ (range, 30-100), and $43 \pm 23$ (range, 20-80), respectively. Of the other 22 neurons, 13 evoked slow EPSPs at $10 \mathrm{~Hz}$ (mean minimum number of APs: $163 \pm 108$; range, 40-450), and 9 did not evoke slow EPSPs until stimulated at $20 \mathrm{~Hz}$ (mean minimum number of APs: $262 \pm 134$; range, 60-500).

When the minimum number of action potentials required to evoke a slow EPSP at $10 \mathrm{~Hz}$ was delivered at $20 \mathrm{~Hz}$, slow EPSP amplitudes and durations underwent mean increases of 64 and $125 \%$, respectively. Doubling the duration of minimally effective $20 \mathrm{~Hz}$ trains caused amplitudes and durations to undergo mean increases of 84 and $208 \%$, respectively. Maximal stimulation at $20 \mathrm{~Hz}$ (>30 sec) evoked slow EPSPs up to $5 \mathrm{~min}$ long. Cholinergic neurons were judged not to be dual function if they failed to evoke detectable slow EPSPs when stimulated at 20 $\mathrm{Hz}$ for $30 \mathrm{sec}$. This criterion may have led to underestimation of the number of dual-function neurons (see Immunocytochemical results, below).

Rundown of slow EPSPS. Figure 2 illustrates the effects on 



Figure 2. Rundown of slow EPSPs. Slow EPSPs were evoked by stimulation of a dual-function neuron for $3 \mathrm{sec}$ at $15 \mathrm{~Hz}$. $A$, The second and third slow EPSPs were evoked 8 and $17 \mathrm{~min}$ after the first one. All 3 slow EPSPs had similar amplitudes. $B$, The first slow EPSP was evoked 12 min after the last one in $A$. The second and third ones were evoked 2 and $4 \mathrm{~min}$ later. Their amplitudes and durations were much smaller. The fourth slow EPSP, evoked $10 \mathrm{~min}$ after the third, had a similar amplitude to the first slow EPSP. The fourth slow EPSP was evoked by a $5 \mathrm{sec}$ train. Calibration bars: $20 \mathrm{mV}, 20 \mathrm{sec}$.

the amplitude of slow EPSPs of varying the interval between trains of presynaptic stimuli. The amplitudes of consecutively evoked slow EPSPs declined substantially unless the postsynaptic membrane potential had returned to resting levels for at least $3 \mathrm{~min}$. However, even when slow EPSPs were evoked at 10 min intervals, their amplitudes tended to decline. This decline in the amplitude of slow EPSPs probably resulted from decreased postsynaptic responsiveness to the slow cotransmitter because responses to repeated applications of VIP declined in a parallel manner. Rundown of submaximal slow EPSPs occurred more slowly than did rundown of maximal slow EPSPs. Accordingly, when testing the effects of experimental manipulations on slow EPSPs, it was convenient to use the minimum presynaptic stimulus that produced an easily detectable slow EPSP.

\section{Comparison of postsynaptic responses to VIP and the noncholinergic cotransmitter}

Figure 3 compares the responses of 2 neurons to VIP and to stimulation of dual-function neurons that innervated them. It also shows that stimulation of a presynaptic neuron during a postsynaptic response to VIP failed to evoke a slow EPSP, presumably because of desensitization of VIP receptors. Although all neurons that received dual synaptic inputs responded to VIP, their sensitivity varied considerably. Some neurons responded readily to $10 \mathrm{nM}$ VIP, while others responded only weakly to concentrations below $1 \mu \mathrm{M}$. Neurons with larger resting conductances tended to be the most sensitive.

Fast EPSP and action potential activity often increased in postsynaptic neurons during responses to VIP. To distinguish direct postsynaptic effects of VIP from indirect effects caused by excitation of terminals that contacted postsynaptic neurons, the effects of extracellular solutions containing reduced $\mathrm{CaCl}_{2}$ ( 0.36 instead of $2.5 \mathrm{~mm}$ ) and $10 \mathrm{~mm} \mathrm{MgCl}_{2}$ were tested. These solutions blocked both components of dual transmission but did not significantly alter slow depolarizations caused by VIP (Fig. 4B).

Conductance changes. Slope conductance decreased during slow EPSPs and during responses to VIP (Fig. 5). The voltagecurrent $(V-I)$ curves obtained from postsynaptic neurons during

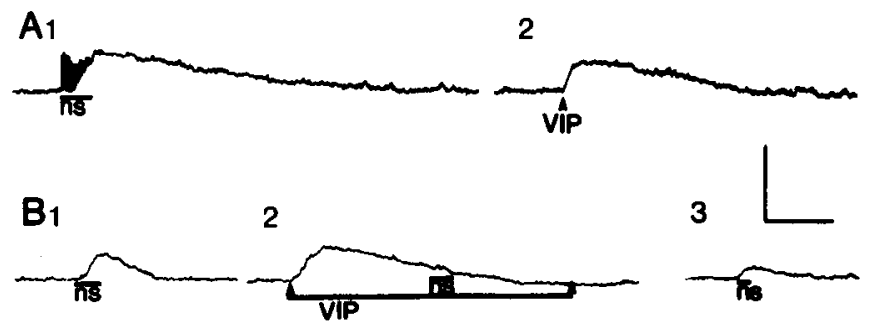

Figure 3. Responses to VIP mimic slow EPSPs evoked by dual-function myenteric neurons. $A: 1$, During the time indicated by the bar labeled $n s$, a presynaptic neuron was stimulated at $10 \mathrm{~Hz}$, evoking nicotinic fast EPSPs and a noncholinergic slow EPSP. 2, Pressure ejection of $100 \mathrm{nM}$ VIP evoked a response that mimicked the slow EPSP. $B: 1$, Stimulation of a different presynaptic neuron in the presence of cholinergic antagonists ( $100 \mu \mathrm{M}$ hexamethonium and $10 \mu \mathrm{M}$ atropine) evoked only a slow EPSP. 2, Stimulation of the presynaptic neuron failed to evoke a slow EPSP during a depolarization evoked by superfusion of $1 \mu \mathrm{M}$ VIP. 3, Five minutes after washing out the VIP, presynaptic stimulation again evoked a slow EPSP. Calibration bars: $20 \mathrm{mV}$, $20 \mathrm{sec}$.

slow EPSPs or during responses to VIP intersected control $\mathrm{V}$ $I$ curves between -80 and $-90 \mathrm{mV}$, near the estimated $\mathrm{K}^{+}$ equilibrium potential of $-84 \mathrm{mV}$. When postsynaptic membrane potential was clamped at its resting level by injection of hyperpolarizing current during a slow EPSP or during a response to VIP, slope conductance decreased for a period of time similar to the duration of the slow EPSP or VIP response in the unclamped cell (Fig. 5B). Conductance decreased $28 \pm 17 \%(n=$ 10) during slow EPSPs of maximal amplitude. The maximal decreases during slow EPSPs were smaller than the maximal changes that could be evoked by VIP. Addition of nicotinic antagonists did not alter the maximal conductance changes observed during slow EPSPs (e.g., Fig. $5 B$ ), making it unlikely that conductances activated during fast EPSPs were partially shunting the effects of the slow transmitter. This suggests either that the amplitudes of the slow EPSPs were limited by the amount of slow cotransmitter that reached the postsynaptic cell or that synaptically released VIP did not have access to the same surface area as did bath applied VIP.

Effects of postsynaptic membrane potential. Injection of progressively larger hyperpolarizing currents into postsynaptic cells caused slow EPSPs (Fig. 6) and responses to VIP (data not shown) to decrease and eventually to reverse polarity. Reversal potentials determined this way did not differ significantly from those predicted by the points of intersection of $V-I$ curves such as those in Figure 5. Slow EPSPs and VIP responses showed no detectable voltage dependence when evoked at membrane potentials between -90 and $-50 \mathrm{mV}$.

Effects of altered concentrations of ions. Altering the concentration of external $\mathrm{K}^{+}$caused significant changes in the reversal potentials of slow EPSPs (Fig. 7 $A$ ) and of responses to VIP (Fig. $7 B$ ). In contrast, altering external $\mathrm{Na}^{+}$or $\mathrm{Cl}^{-}$concentrations had very little effect (data not shown). The reversal potentials of VIP responses and of slow EPSPs changed $54 \pm 6(n=8)$ and 57 $\pm 3(n=9) \mathrm{mV}$ per 10 -fold change in $[\mathrm{K}]_{\mathrm{o}}$, respectively. These changes are close to the $58 \mathrm{mV}$ per 10 -fold change predicted by the Nernst equation.

\section{Effects of antisera on slow EPSPS}

The data in the preceding sections are consistent with the hypothesis that VIP is a cotransmitter of dual-function myenteric 

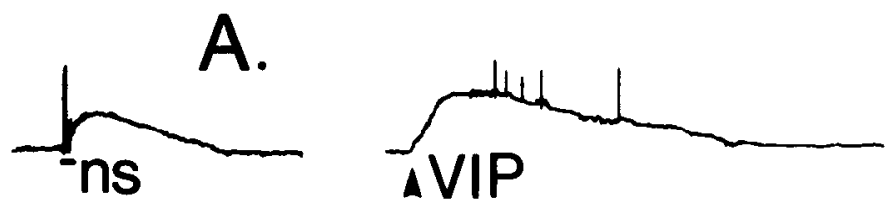

B.
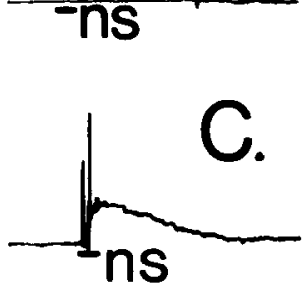
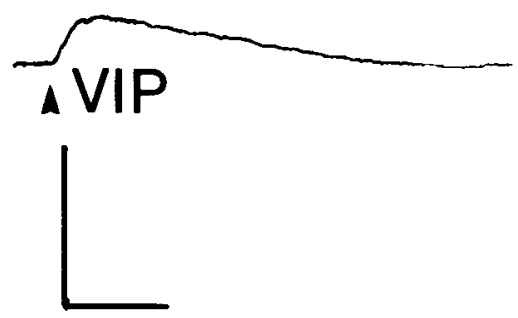

Figure 4. Reducing extracellular Ca blocks synaptic transmission but not responses to VIP. $A$, The response to pressure ejection of $50 \mathrm{~nm}$ VIP (right trace) mimicked the slow EPSP evoked by stimulation of a dual-function neuron (left trace). Five fast EPSPs occurred during the VIP response. $B$, The response to VIP persisted in a low-Ca, high- $\mathrm{Mg}$ solution that prevented both fast and slow EPSPs. $C$, Both fast and slow EPSPs recovered following return to normal concentrations of divalent cations. Calibration bars: $20 \mathrm{mV}, 20 \mathrm{sec}$.

neurons. However, responses of some postsynaptic neurons to another enteric neurotransmitter candidate, substance $P$, also mimicked the slow EPSPs (data not shown). Furthermore, desensitization to substance $P$ prevented slow EPSPs evoked by 4 of 11 dual-function neurons tested. This result substantially weakens the strength of arguments based upon VIP's ability to block slow EPSPs. For example, the ability of VIP to block slow EPSPs might result not from desensitization, but rather from closure of the same set of channels normally closed by the authentic transmitter. A precedent for such a phenomenon is the ability of bethanechol, a cholinergic agonist, to prevent the responses of bullfrog sympathetic neurons to LHRH (Kuffler and Sejnowski, 1983). Thus, in order to argue that VIP is the slow cotransmitter, it is necessary but not sufficient to demonstrate that VIP can mimic and block slow EPSPs evoked by dualfunction neurons. Although dual-function neurons contain IRVIP and do not contain detectable amounts of SP-LIR (Willard and Nishi, 1987), it is possible that they do not release VIP in response to stimuli that cause slow EPSPs. Rather, they might release a different molecule with similar postsynaptic actions.

To test the hypothesis that the authentic slow cotransmitter is VIP, it is necessary to have specific antagonists for VIP receptors. One strategy that has been used successfully in several studies of peptidergic transmission is to use antisera to prevent peptide molecules from reaching their postsynaptic receptors. Anti-VIP antisera reduce or prevent neurally evoked responses in several systems where VIP is thought to act as a slow transmitter (Goyal et al., 1980; Lundberg et al., 1980; Angel et al., 1984; Grider et al., 1985; Grider and Makhlouf, 1986; Love and Szurszewski, 1987). Accordingly, the ability of bath application of VIP antisera to alter the slow EPSPs evoked by dualfunction neurons was tested. As illustrated in Figure 8, anti-VIP antiserum reversibly and selectively antagonized the slow EPSP. The antagonistic effects occurred within $5 \mathrm{~min}$ of application

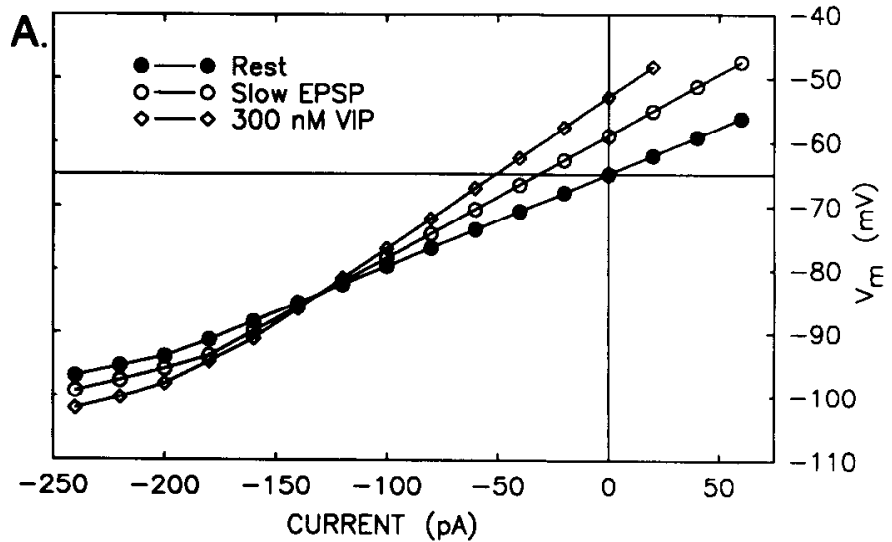

B.

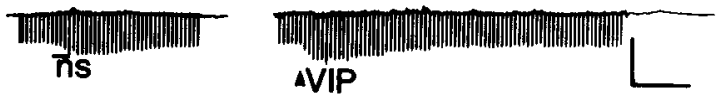

Figure 5. Comparison of conductance changes during slow EPSPs and responses to VIP. $A$, Voltage-current $(V-I)$ relations of a myenteric neuron at rest (solid circles), during a slow EPSP evoked by a dualfunction neuron (open circles), and during a response to bath application of $300 \mathrm{~nm}$ VIP (open diamonds) were determined by measuring voltage changes evoked by injection of pulses of current. Both the slow EPSP and VIP $V-I$ curves intersect the control $V-I$ curve at approximately $-85 \mathrm{mV}$, which is very close to the estimated $\mathrm{K}^{+}$equilibrium potential of $-84 \mathrm{mV}$. $B$, Hyperpolarizing current pulses caused larger voltage changes during a manually clamped slow EPSP (left trace) and during a manually clamped response to puffer application of $100 \mathrm{nM}$ VIP (right trace). Hexamethonium $(50 \mu \mathrm{M})$ and atropine $(10 \mu \mathrm{M})$ were present to prevent any actions of ACh. Calibration bars: $20 \mathrm{mV}, 20 \mathrm{sec}$.

(shorter times were not tested in order to minimize rundown). Complete recovery occurred following $10 \mathrm{~min}$ of washing (approximately 10 complete exchanges of the bath volume). These relatively rapid and reversible effects of antisera suggest that the sites of VIP release are readily accessible even to large macromolecules in extracellular solutions. Three different antisera raised against 3 different conjugates of VIP gave similar results. All 3 antisera were tested at dilutions of 1:10, 1:20, 1:50, 1:100, and 1:200. All 3 antisera blocked slow EPSPs at 1:10 and 1:20. Antiserum $\mathrm{H}$ also blocked at 1:50 and caused decreases at 1:100 and 1:200. Antiserum $M$ caused decreases at 1:50 and 1:100 but had no reliable effects at 1:200. Antiserum $S$ was the weakest, having no significant effects at 1:100. Normal rabbit sera diluted $1: 10$ and anti-substance $P$ antisera at $1: 10$ or $1: 20$ had no significant effects on the slow EPSPs evoked by dual-function neurons.

Anti-VIP antisera did not cause significant changes in resting membrane potential, action potential amplitude, action potential duration, or input resistance of presynaptic cell bodies $(n=$ 6; data not shown). Figure 8 also illustrates that anti-VIP did not cause detectable changes in the amplitude of fast nicotinic EPSPs evoked by low-frequency stimulation of dual-function neurons $(n=9)$. I interpret these results to mean that anti-VIP antagonized slow EPSPs by binding released VIP and preventing it from interacting with its postsynaptic receptors rather than by preventing release of VIP from the presynaptic neurons or by blocking postsynaptic receptors.

\section{Immunocytochemical results}

After electrophysiological experiments, cultures were switched from recording solution to serum-free medium and placed in 


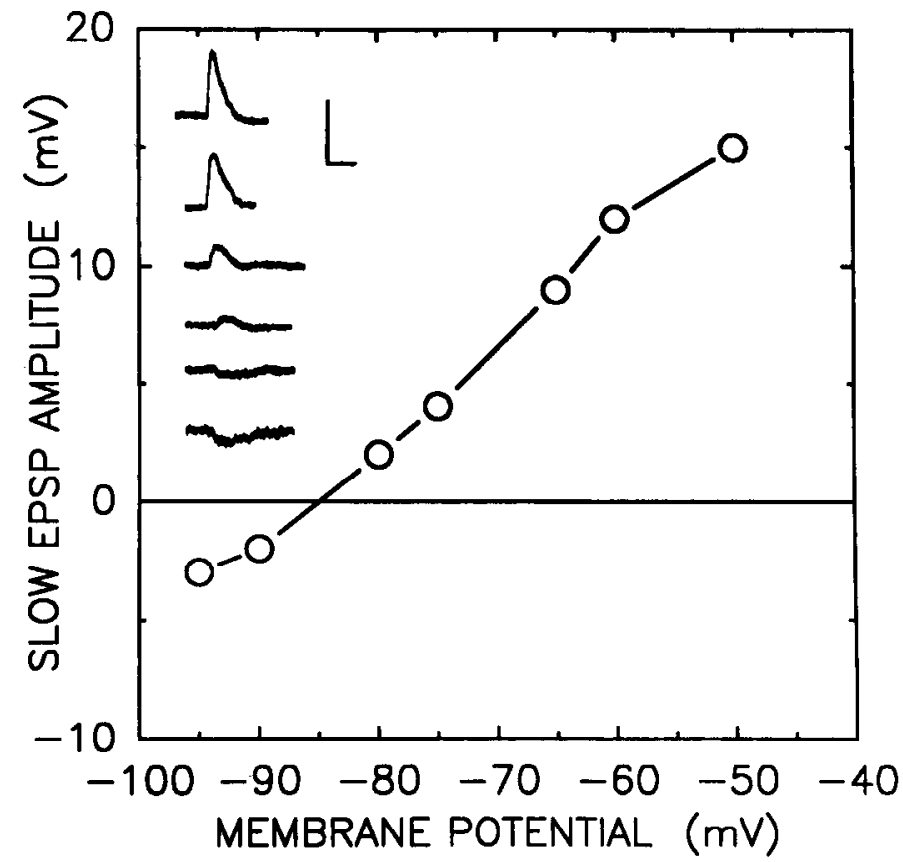

Figure 6. Effect of altered postsynaptic membrane potential on amplitudes of slow EPSPs. Insets show slow EPSPs evoked by stimulation of a dual-function neuron for $2 \mathrm{sec}$ at $10 \mathrm{~Hz}$. Hexamethonium $(50 \mu \mathrm{M})$ and atropine $(10 \mu \mathrm{M})$ were present to block the actions of $\mathrm{ACh}$. The graph shows that the estimated reversal potential is $-86 \mathrm{mV}$, close to the estimated $\mathrm{K}^{+}$equilibrium potential of $-84 \mathrm{mV}$. Calibration bars inset: $15 \mathrm{mV}, 20 \mathrm{sec}$.

the incubator for several hours before being fixed and stained for VIP-LIR. Seventeen of the 42 dual-function neurons in this study were relocated successfully after immunocytochemical processing of the cultures. All 17 (100\%) were VIP-positive, thereby confirming and extending the finding of Willard and Nishi (1987) that dual-function neurons contain VIP-LIR. In contrast, VIP-LIR was detected in only $9 \%(18 / 207)$ of physiologically monofunctional neurons that evoked only fast cholinergic EPSPs. There are several possible explanations for the observation of VIP-LIR in apparently monofunctional cholinergic neurons: (1) some ACh/VIP neurons release too little VIP to cause detectable slow EPSPs; (2) some postsynaptic neurons are insensitive to VIP (or do not have VIP receptors near the site of release); (3) ACh/VIP neurons do not release VIP at all their synapses; or (4) some ACh/VIP neurons release cotransmitters onto postsynaptic sites that are sufficiently electrotonically distant from the cell body that only the fast EPSPs can be detected by recordings from cell bodies of postsynaptic neurons. The total proportion of VIP-positive neurons in cultures that had been used for electrophysiological experiments $(32 \pm 9 \%$; $n=31$ ) did not differ significantly from that of control cultures $(30 \pm 7 \% ; n=35)$.

\section{Discussion}

The use of cell culture has permitted the first analysis of cotransmission between mammalian neurons at the level of individual pairs of pre- and posisynaptic neurons. I will discuss first the synaptic actions of the noncholinergic cotransmitter and the evidence suggesting it is VIP. I will then compare these results to other studies of peptidergic cotransmission.

\section{A. SLOW EPSPs}

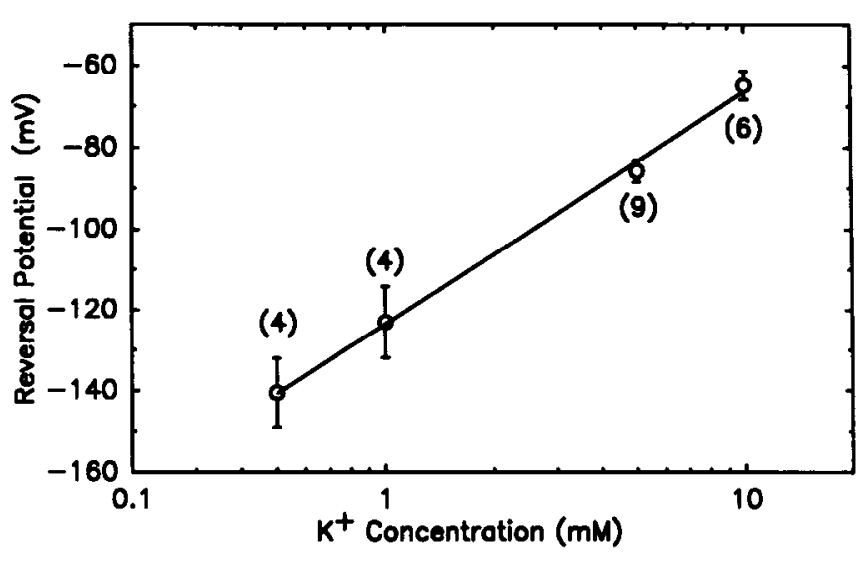

B. VIP RESPONSES

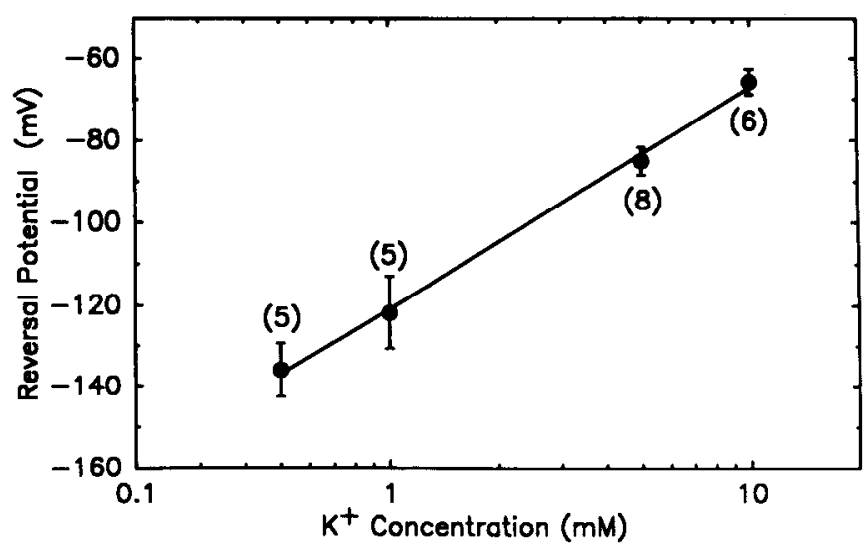

Figure 7. Effects of altered extracellular $\mathrm{K}^{+}$on the reversal potential of slow EPSPs $(A)$ and responses to VIP $(B)$. Reversal potentials were estimated from the points of intersection of $V-I$ curves of neurons at rest and during slow EPSPs. The slopes of the regression lines in $A$ and $B$ are 57 and $54 \mathrm{mV}$ per 10 -fold change in $\mathrm{K}^{+}$concentration. The error bars are SDs. Numbers in parentheses are the number of neurons tested for each point.

Properties of slow EPSPs evoked by dual-function myenteric neurons

Identification of the slow cotransmitter. This study confirmed and extended the finding of Willard and Nishi (1987) that dualfunction myenteric neurons contain immunoreactive VIP. The following electrophysiological and pharmacological data argue that a VIP-like molecule mediates slow EPSPs evoked by stimulation of dual-function myenteric neurons. (1) Exogenously applied VIP mimicked the slow noncholinergic EPSPs. Responses to VIP causcd similar conductance changes, reversed at the same membrane potential and had the same ionic dependence as did the slow EPSPs. (2) Desensitization of postsynaptic neurons to VIP prevented slow EPSPs and responses to VIP were reduced or prevented by slow EPSPs. (3) Antisera directed against VIP reduced or prevented the slow EPSPs selectively and reversibly. I have suggested that the antisera antagonized slow EPSPs because antibodies bound released VIP and prevented it from interacting with its postsynaptic receptors. An alternative possibility is antagonism resulting from binding of antibodies to cell surface molecules necessary for release of 
or responsiveness to the slow cotransmitter. I consider this possibility unlikely because the antisera caused no discernable changes in the properties of the presynaptic neurons, including their ability to cause fast EPSPs. Furthcrmorc, binding of antibodies to cell surface components should not be readily reversible. For example, binding of antibodies to postsynaptic receptors (e.g., Stollberg et al., 1986) or to presynaptic Ca channels (e.g., Kim and Neher, 1988) would probably cause effectively irreversible blockade of the slow EPSP.

The strength of the immunological data depends on the specificity of the antisera used. Because antisera that were raised against 3 different conjugates of VIP gave similar results, it is likely that the slow cotransmitter is either authentic VIP or a closely related molecule. However, precise molecular identification of the slow cotransmitter awaits detailed biochemical analysis of the VIP-LIR contained in and released by cultures of myenteric neurons.

Postsynaptic mechanism of slow EPSPS. Three lines of evidence support the hypothesis that decreased resting $\mathrm{K}^{+}$conductance causes the slow EPSPs. (1) Slope resistance of postsynaptic neurons increased during slow EPSPs. (2) Slow EPSPs reversed polarity at or near the calculated $\mathrm{K}^{+}$equilibrium potential. In solutions containing altered concentrations of $\mathrm{K}^{+}$, the reversal potential shifted as predicted by the Nernst equation. (3) The linear voltage-current relation between -90 and -50 $\mathrm{mV}$ argues that suppression of voltage-dependent channels such as inward rectifiers (Stanfield et al., 1985) or M-channels (Adams and Brown, 1982) did not cause the slow EPSPs. It seems more likely that the slow cotransmitter suppressed a resting $\mathrm{K}^{+}$conductance. For example, synaptically mediated decreases of resting $\mathrm{K}^{+}$conductances occur in guinea pig myenteric neurons (Johnson et al., 1980) and in Aplysia sensory neurons (Klein et al., 1982).

The actions of VIP and the slow cotransmitter resemble those observed by Zafirov et al. (1985), who found that VIP increases input resistance of guinea pig myenteric neurons, and by Akasu et al. (1986), who found that VIP reduces a voltage-insensitive $\mathrm{K}^{+}$conductance in parasympathetic neurons of the cat bladder. In contrast, VIP causes decreased membrane resistance in rat dorsal horn neurons (Jeftinija et al., 1982) and carp retinal horizontal cells (Lasater et al., 1983) and either increased or decreased resistance of guinea pig sympathetic neurons (Love and Szurszewski, 1987). Thus, VIP can act via a variety of biophysical mechanisms.

Further experiments will be needed to discover the mechanism(s) by which VIP decreases $\mathrm{K}^{+}$conductance. One likely mechanism would be activation of adenylate cyclase. VIP causes increased synthesis of cyclic AMP in many cell types (for references, see Said and Mutt, 1988). Alterations in levels of cyclic AMP modulate at least 2 different $\mathrm{K}^{+}$conductances in Aplysia neurons, including a resting $\mathrm{K}^{+}$conductance that resembles the one modulated by the slow cotransmitter of dual-function myenteric neurons (Siegelbaum et al., 1982; Walsh and Byrne, 1989). Furthermore, in guinea pig myenteric neurons, agents that increase adenylate cyclase activity mimic the effects of VIP and agents that interfere with adenylate cyclase activity prevent responses to VIP (Palmer et al., 1987).

\section{Comparison to other cases of cotransmission}

Requirement for multiple stimuli to evoke slow EPSPS. Dualfunction myenteric neurons evoked slow EPSPs only in response to multiple stimuli. Previous studies of cotransmission have

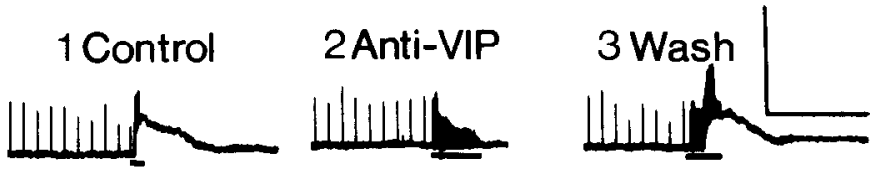

Figure 8. Antagonism of slow EPSPs by anti-VIP. 1, Presynaptic stimulation at $0.2 \mathrm{~Hz}$ evoked only fast EPSPs, which are seen as rapid upward deflections. Increasing the frequency of stimulation to $8 \mathrm{~Hz}$, during the time indicated by the bar beneath the trace, evoked a slow EPSP. 2, When the culture was bathed in anti-VIP (antiserum $\mathrm{H}$ diluted 1:50), $8 \mathrm{~Hz}$ stimulation failed to evoke a slow EPSP. During the high-frequency train, the amplitudes of the fast EPSPs declined significantly. 3, Following washout of the anti-VIP antiserum, $8 \mathrm{~Hz}$ stimulation again evoked a slow F.PSP. Calibration hars: $20 \mathrm{mV}, 36 \mathrm{sec}$

made similar findings: single presynaptic action potentials reliably elicit postsynaptic responses caused by classical transmitters, but multiple presynaptic stimuli are required to elicit slower, peptide-mediated postsynaptic responses (Lundburg et al., 1980; Jan and Jan, 1982; Jan et al., 1983; Bishop et al., 1987; Li and Calabrese, 1987; Bartfai et al., 1988; Lundberg et al., 1989). The simplest explanation of the requirement for multiple stimuli is that single action potentials release insufficient amounts of VIP to cause detectable postsynaptic responses. This explanation seems especially plausible for synapses in cell cultures, where there is likely to be rapid dilution of neurally released substances. In freshly dissected myenteric plexus, multiple stimuli are usually necessary to evoke slow EPSPs, but single stimuli to nerve bundles sometimes evoke noncholinergic slow EPSPs (Morita and North, 1985; Brookes et al., 1988). It is not known whether the slow EPSPs evoked by such stimuli result from activation of more than one presynaptic neuron, nor is it known whether any of the neurons activated by such stimuli are dual-function neurons.

The requirement for multiple stimuli, along with the apparent decrease in release of $\mathrm{ACh}$ during trains of stimuli that evoked slow EPSPs, suggests that separate mechanisms control release of $\mathrm{ACh}$ and the cotransmitter. A likely basis for independent release of ACh and VIP is storage in and release from different types of secretory vesicles. Many peptides are stored in densecore vesicles (e.g., Lundberg et al., 1981; Probert et al., 1983), whereas ACh is generally believed to be stored in small synaptic vesicles. Another possibility is that during low-frequency stimulation, $\mathrm{ACh}$ binds to muscarinic receptors on the presynaptic terminals and inhibits release of VIP sufficiently to prevent slow EPSPs. Morita et al. (1982) showed that muscarinic antagonists partially prevented the frequency-dependent rundown of fast EPSPs and that they enhanced slow noncholinergic EPSPs. In cultures of myenteric neurons, however, atropine, which does not antagonize the slow EPSPs evoked by dual-function neurons (Figs. 3,6), does not reduce the number of stimuli required to evoke them either (Willard and Nishi, 1985b, 1987, and A. L. Willard, unpublished observations). Thus, it appears unlikely that muscarinic inhibition accounts for the frequency-dependence of slow EPSPs.

Calcium dependence of $V I P$ release. Solutions containing lowered $\mathrm{Ca}$ and $10 \mathrm{~mm} \mathrm{Mg}$ (Fig. $4 B$ ) blocked all postsynaptic effects of dual-function neurons. This blockade most likely resulted from decreased influx of Ca into presynaptic terminals. Release of peptide cotransmitters is Ca-dependent in bullfrog sympathetic ganglia (Jan et al., 1979; Jan and Jan, 1982) and at cockroach neuromuscular junction (Adams and O'Shea, 1983). Sim- 
ilarly, release of substance P from sensory neurons (Holz et al., 1988), from parasympathetic neurons (Bowers et al., 1986), and from enteric neurons (Baron et al., 1983; Holzer, 1984) requires extracellular calcium. Howcver, it has bccn reported that electrically evoked release of VIP from rat ileum does not require extracellular Ca (Belai et al., 1987). Thus, the mechanisms hy which neuronal electrical activity triggers release of peptide cotransmitters, and the relationship between intracellular Ca levels and secretion of peptide cotransmitters require further investigation.

Possible physiological significance of dual transmission. The VIP-like cotransmitter released by dual-function myenteric neurons directly excited postsynaptic neurons by depolarizing them, often causing them to fire action potentials. It also indirectly excited them because the increased postsynaptic input resistance caused subthreshold EPSPs to become suprathreshold. This agrees with the finding of Williams and North (1979) that VIP increases the rate of firing detected by extracellular electrodes placed on myenteric ganglia. It also supports the results of several pharmacological studies that suggested VIP excites cholinergic and other myenteric neurons (e.g., Yau et al., 1985; Kusonoki et al., 1986). The effects of the cotransmitter may also help compensate for the decreased amplitudes of fast EPSPs that occur when cholinergic myenteric neurons are stimulated at moderate or high frequencies.

In other systems, peptide cotransmitters have either direct or modulatory actions on postsynaptic cells. In bullfrog sympathetic ganglia, an LHRH-like peptide mediates slow synaptic excitation (Jan et al., 1979, 1983; Jan and Jan, 1982) and in leech heart a FMRFamide-like peptide causes increased myogenic activity (Li and Calabrese, 1987). Both of these substances are released from cholinergic neurons and have postsynaptic actions easily seen in the absence of cholinergic transmission. Other peptide cotransmitters modulate the effects of their classical cotransmitters without causing detectable responses by themselves. For example, at the crayfish neuromuscular junction, proctolin enhances contractions caused by its fast cotransmitter glutamate but has no observable effects on the muscle in the absence of nerve stimulation (Bishop et al., 1987).

In the cat salivary gland, VIP has both direct effects, causing atropine-resistant vasodilatation, and modulatory effects, enhancing the actions of $\mathrm{ACh}$ by increasing the affinity of muscarinic receptors (Lundberg et al., 1980, 1982). VIP also cnhances muscarinic responses of guinea pig sympathetic neurons (Mo and Dun, 1984) and of cat sympathetic and parasympathetic neurons (Kawatani et al., 1985a, b). It is not known if VIP enhances muscarinic transmission in myenteric ganglia. Muscarinic synaptic potentials occur in guinea pig myenteric neurons (North and Tokimasa, 1982) but were not observed in rat myenteric plexus by Brookes et al. (1988). Unfortunately, the postsynaptic neurons studied did not have detectable muscarinic responses to $\mathrm{ACh}$ (see also Willard and Nishi, 1985a), thereby precluding a test of whether VIP enhanced muscarinic responses. It is possible that different culture conditions, under which significant numbers of myenteric neurons do have muscarinic responses to ACh (Nishi and Willard, 1988), will permit tests for interactions between VIP and muscarinic receptors.

\section{Do ACh/VIP myenteric neurons occur in vivo?}

Although many enteric neurons are cholinergic and most enteric neurons contain one or more peptidelike immunoreactivities (e.g., Furness and Costa, 1987), there are no physiological data that address directly the question of whether $\mathrm{ACh} / \mathrm{VIP}$ dualfunction neurons occur in rat myenteric plexus in vivo. However, in a study of myenteric plexus of rat duodenum, Brookes et al. (1988) observed that slowly increasing the strength of stimuli delivered to nerve bundles evoked additional fast and slow EPSPs at exactly the same thresholds. This suggests that the fast and slow EPSPs were evoked by neurons with the same thresholds and is consistent with the hypothesis that some cholinergic myenteric interneurons release a slow cotransmitter. Dual-function interneurons might also project to prevertebral sympathetic neurons. Love and Szurszewski (1987) found that stimulation of nerves projecting from the colon to the inferior mesenteric ganglia in guinea pigs evoked both fast nicotinic EPSPs and slow noncholinergic EPSPs that were partially antagonized by antiVIP. Thus, it is possible that they were stimulating ACh/VIP dual-function neurons.

It is also possible that the dual-function neurons observed in cell culture normally project to non-neuronal targets such as smooth muscles or blood vessels in vivo. It seems less likely that ACh/VIP mycntcric neurons would be motor neurons because ACh and VIP generally have opposing actions on intestinal smooth muscle (e.g., Grider et al., 1985; Grider and Makhlouf, 1986). However, ACh/VIP neurons contact blood vessels in a variety of tissues (Lundberg et al., 1980; Feher and Leranth, 1983; Eckenstein and Baughman, 1984).

Ultrastructural analysis of VIP-containing neurons in rat myenteric plexus reveals both small $(35-40 \mathrm{~nm})$ clear and larger $(80-120 \mathrm{~nm})$ dense-core vesicles (Feher and Leranth, 1983). Such observations are compatible with colocalization of VIP and a classical cotransmitter. However, to date there have been no published reports of direct immunocytochemical evidence for colocalization of VIP and choline acetyltransferase in rat myenteric neurons. Most if not all VIP-positive neurons in rat myenteric plexus do contain immunoreactive peptide histidineisoleucine (a product of prepro-VIP) and neuropeptide Y (Ekblad et al., 1984, 1988). If any of these neurons arc also cholinergic, their postsynaptic actions could be quite complex. The pharmacological actions of PHI on myenteric neurons are unknown, but NPY generally has inhibitory pharmacological actions on myenteric neurons (Garzon et al., 1986; Hirning et al., 1988). Whatever their in vivo function may be, dual-function neurons in cell cultures should be useful for future studies of the rcgulation of release of cotransmitters.

\section{References}

Adams, M., and M. O'Shea (1983) Peptide co-transmitter at neuromuscular junction. Science 221: 286-289.

Adams, P., and D. Brown (1982) Synaptic inhibition of the M-current: Slow excitatory postsynaptic potential mechanism in bullfrog sympathetic neurons. J. Physiol. (Lond.) 332: 263-272.

Akasu, T., J. P. Gallagher, K. Hirai, and P. Shinnick-Gallagher (1986) VIP depolarizations in cat bladder parasympathetic ganglia. J. Physiol. (Lond.) 374: 457-473.

Angel, F., V. Go, and J. Szurszewski (1984) Innervation of the muscularis mucosae of canine proximal colon. J. Physiol. (Lond.) 357 . 93-108.

Baron, S., B. M. Jaffe, and A. Gintzler (1983) Release of Substance P from the enteric nervous system: Direct quantitation and characterization. J. Pharmacol. Exp. Ther. 227: 365-368.

Bartfai, T., K. Iverfeldt, G. Fisone, and P. Serfozo (1988) Regulation of the release of coexisting neurotransmitters. Annu. Rev. Pharmacol. Toxicol. 28: 285-310.

Belai, A., V. Ralevic, and G. Burnstock (1987) VIP release from enteric nerves is independent of extracellular calcium. Reg. Peptides 19:7989. 
Bishop, C., J. Wine, F. Nagy, and M. O'Shea (1987) Physiological consequences of a peptide cotransmitter in a crayfish nerve-muscle preparation. J. Neurosci. 7: 1769-1779.

Bornstein, J. C., R. A. North, M. Costa, and J. B. Furness (1984) Excitatory synaptic potentials due to activation of neurons with short projections in the myenteric plexus. Neuroscience 11: 723-731.

Bowers, C. W., L. Y. Jan, and Y. N. Jan (1986) A Substance P-like peptide in bullfrog autonomic nerve terminals: Anatomy, biochemistry and physiology. Neuroscience 19: 343-356.

Brookes, S. J. H., W. R. Ewart, and D. L. Wingate (1988) Intracellular recordings from cells in the myenteric plexus of the rat duodenum. Neuroscience 24: 297-307.

Dreyer, S. E., and V. A. Chiappinelli (1985) Properties of choroid and ciliary neurons in the avian ciliary ganglion and evidence for substance $P$ as a neurotransmitter. J. Neurosci. 5: 2654-2661.

Eckenstein, F., and R. W. Baughman (1984) Two types of cholinergic innervation in cortex, one colocalized with VIP. Nature 309: 153155.

Ekblad, E., R. Hakanson, and F. Sundler (1984) VIP and PHI coexist with an NPY-like peptide in intramural neurones of the small intestine. Reg. Peptides 10: 47-55.

Ekblad, E., R. Ekman, R. Hakanson, and F. Sundler (1988) Projections of peptide-containing neurons in rat colon. Neuroscience 27:655674.

Feher, E., and C. Leranth (1983) Light and electron microscopic immunocytochemical localization of VIP-like activity in the rat small intestine. Neuroscience 10:97-106.

Furness, J. B., and M. Costa (1987) The Enteric Nervous System, Churchill Livingstone, New York.

Garzon, J., V. Hollt, and P. Sanchez-Blazquez (1986) Neuropeptide $\mathrm{Y}$ is an inhibitor of neural function in the myenteric plexus of the guinea-pig ileum. Peptides 7: 623-629.

Goyal, R. K., S. Rattan, and S. I. Said (1980) VIP as a possible neurotransmitter of non-cholinergic non-adrenergic inhibitory neurones. Nature 288: 378-380.

Grider, J. R., and G. M. Makhlouf (1986) Colonic peristaltic reflex: Identification of vasoactive intestinal peptide as mediator of descending relaxation. Am. J. Physiol. 25 I (Gastrointest. Liver Physiol. 14): G40-G45.

Grider, J. R., M. B. Cable, K. N. Bitar, S. I. Said, and G. M. Makhlouf (1985) Vasoactive intestinal peptide. Relaxant neurotransmitter in tenia coli of the guinea pig. Gastroenterology 89: 35-42.

Hamill, O., A. Marty, E. Neher, B. Sakmann, and F. Sigworth (1981) Improved patch clamp techniques for high-resolution current recording from cells and cell-free membrane patches. Pfluegers Arch. 391: 85-100.

Hanani, M., and G. Burnstock (1985) Synaptic activity of myenteric neurons in tissue culture. J. Autonomic Nervous System 14:49-60.

Hirano, T., Y. Kubo, and M. M. Wu (1986) Cerebellar granule cells in culture: Monosynaptic connections with Purkinje cells and ionic currents. Proc. Natl. Acad. Sci. USA 83: 4957-4961.

Hirning, L., R. Miller, and A. Fox (1988) Modulation of $\mathrm{Ca}^{2+}$ transients and $\mathrm{Ca}^{2+}$ currents in cultured rat myenteric neurons by Neuropeptide Y. Biophys. J. 53: 234a.

Hökfelt, T., D. Millhorn, K. Seroogy, Y. Tsuruo, S. Ceccatelli, B. Lindh, B. Meister, T. Melander, M. Schalling, T. Bartfai, and L. Terenius (1987) Coexistence of peptides with classical neurotransmitters. Experientia 43: 768-780.

Holz, G., K. Dunlap, and R. Kream (1988) Characterization of the electrically evoked release of Substance P from DRG neurons: Methods and dihydropyridine sensitivity. J. Neurosci. 8: 463-471.

Holzer, P. (1984) Characterization of the stimulus-induced release of immunoreactive Substance $P$ from the myenteric plexus of the guineapig small intestine. Brain Res. 297: 127-136.

Jan, L. Y., and Y. N. Jan (1982) Peptidergic transmission in sympathetic ganglia of the frog. J. Physiol. (Lond.) 327: 219-246.

Jan, Y. N., L. Y. Jan, and S. W. Kuffler (1979) A peptide as a possible transmitter in sympathetic ganglia of the frog. Proc. Natl. Acad. Sci. USA 76: 1501-1505.

Jan, Y. N., C. W. Bowers, D. Branton, L. Evans, and L. Y. Jan (1983) Peptides in neuronal function: Studies using frog sympathetic ganglia. Cold Spring Harbor Symp. Quant. Biol. 48: 363-373.

Jeftinija, S., K. Murase, V. Nedeljkov, and M. Randic (1982) VIP excites mammalian dorsal horn neurons both in vivo and in vitro. Brain Res. 243: 158-164.
Johnson, S. M., Y. Katayama, and R. A. North (1980) Slow synaptic potentials in neurones of the myenteric plexus. J. Physiol. (Lond.) 301: 505-516.

Kawatani, M., M. Rutigliano, and W. C. DeGroat (1985a) Depolarization and muscarinic excitation induced in a sympathetic ganglion by vasoactive intestinal polypeptide. Science $229: 879-881$.

Kawatani, M., M. Rutigliano, and W. C. DeGroat (1985b) Selective facilitatory effect of VIP on muscarinic firing in vesical ganglia of the cat. Brain Res. 336: 223-234.

Kim, Y., and E. Neher (1988) IgG from patients with Eaton-Lambert syndrome blocks voltage-dependent calcium currents. Science 239 : 405-408.

Klein, M., J. Camardo, and E. R. Kandel (1982) Serotonin modulates a specific potassium current in the sensory neurons that show presynaptic facilitation in Aplysia. Proc. Natl. Acad. Sci. USA 79: 57135717

Kuffler, S. W., and T. J. Sejnowski (1983) Peptidergic and muscarinic excitation at amphibian sympathetic synapses. J. Physiol. (Lond.) 341: $257-278$.

Kusunoki, M., L. H. Tsai, K. Taniyama, and C. Tanaka (1986) Vasoactive intestinal polypeptide provokes acetylcholine release from the myenteric plexus. Am. J. Physiol. 251 (Gastrointest. Liver Physiol. 14): G51-G55.

Lasater, E. M., K. J. Watling, and J. E. Dowling (1983) VIP alters membrane potential and cyclic nucleotide levels in retinal horizontal cells. Science 221: 1070-1072.

Li, C., and R. Calabrese (1987) FMRFamide-like substances in the leech. III. Biochemical characterization and physiological effects. J. Neurosci. 7: 595-603.

Love, J., and J. Szurszewski (1987) The electrophysiological effects of VIP in the guinea pig inferior mesenteric ganglion. J. Physiol. (Lond.) 394: 67-84.

Lundberg, J. M., A. Anggard, J. Fahrenkrug, T. Hökfelt, and V. Mutt (1980) VIP in cholinergic neurons of exocrine glands: Functional significance of coexisting transmitter for vasodilation and secretion. Proc. Natl. Acad. Sci. USA 77: 1651-1655.

Lundberg, J. M., G. Fried, J. Fahrenkrug, B. Holmstedt, T. Hökfelt, H. Lagerkrantz, G. Lundgren, and A. Anggard (1981) Subcellular fractionation of cat submandibular gland: Comparative studies on the distribution of ACh and VIP. Neuroscience 6: 1001-1010.

Lundberg, J. M., B. Hedlund, and T. Bartfai (1982) VIP enhances muscarinic ligand binding in cat submandibular salivary gland. $\mathrm{Na}$ ture 295: 147-149.

Lundberg, J. M., A. Rudehill, A. Sollevi, G. Fried, and G. Wallin (1989) Co-release of Neuropeptide $Y$ and noradrenaline from pig spleen in vivo: Importance of subcellular storage, nerve impulse frequency and pattern, feedback regulation and resupply by axonal transport. Neuroscience $28: 475-486$.

Matsumoto, S. G., D. Sah, D. D. Potter, and E. J. Furshpan (1987) Synaptic functions in rat sympathetic neuron microcultures. IV. Nonadrenergic excitation of cardiac myocytes and the variety of multipletransmitter states. J. Neurosci. 7: 380-390.

Mihara, S., Y. Katayama, and S. Nishi (1985) Slow postsynaptic potentials in neurones of submucous plexus of guinea-pig caecum and their mimicry by noradrenaline and various peptides. Neuroscience 16: $1057-1068$.

Mo, N., and N. J. Dun (1984) VIP facilitates muscarinic transmission in mammalian sympathetic ganglia. Neurosci. Lett. 52: 19-23.

Morita, K., and R. A. North (1985) Significance of slow synaptic potentials for transmission of excitation in the guinea-pig myenteric plexus. Neuroscience 14: 661-672.

Morita, K., R. A. North, and T. Tokimasa (1982) Muscarinic presynaptic inhibition of synaptic transmission in myenteric plexus of guinea-pig ileum. J. Physiol. (Lond.) 333: 141-149.

Neild, T. O. (1978) Slowly-developing depolarization of neurones in the guinea-pig inferior mesenteric ganglion following repetitive stimulation of the preganglionic nerves. Brain Res. 140:231-239.

Nelson, P., K. Marshall, R. Pun, C. Christian, W. Sheriff, R. MacDonald, and E. Neale (1983) Synaptic interactions between mammalian central neurons in cell culture. II. Quantal analysis of EPSPs. J. Neurophysiol. 49: 1442-1458.

Nelson, P., R. Pun, and G. Westbrook (1986) Synaptic excitation in cultures of mouse spinal cord neurones: Receptor pharmacology and behaviour of synaptic currents. J. Physiol. (Lond.) 372: 169-190.

Nishi, R., and A. L. Willard (1985) Neurons dissociated from rat 
myenteric plexus retain differentiated properties when grown in cell culture. I. Morphological properties and immunocytochemical localization of transmitter candidates. Neuroscience 16: 187-199.

Nishi, R., and A. L. Willard (1988) Conditioned medium alters electrophysiological and transmitter-related properties expressed by rat enteric neurons in cell culture. Neuroscience 25: 759-769.

Nishi, S., and K. Koketsu (1968) Early and late afterdischarges of amphibian sympathetic ganglion cells. J. Neurophysiol. 31: 109-121.

Nishi, S., and R. A. North (1973) Intracellular recording from the myenteric plexus of the guinea-pig ileum. J. Physiol. (Lond.) 231: $471-491$.

North, R. A., and T. Tokimasa (1982) Muscarinic synaptic potentials in guinea-pig myenteric plexus neurones. J. Physiol. (Lond.) 333:151156.

Nusbaum, M. P., and E. Marder (1989) A modulatory proctolincontaining neuron (MPN). II. State-dependent modulation of rhythmic motor activity. J. Neurosci. 9: 1600-1607.

O'Brien, R., and G. D. Fischbach (1986) Excitatory synaptic transmission between interneurons and motoneurons in chick spinal cord cell cultures. J. Neurosci. 6: 3284-3289.

O'Lague, P., D. D. Potter, and E. J. Furshpan (1978) Studies on rat sympathetic neurons developing in cell culture. III. Cholinergic transmission. Dev. Biol. 67: 424-443.

Palmer, J. M., J. D. Wood, and D. H. Zafirov (1987) Transduction of aminergic and peptidergic signals in enteric neurons of the guineapig. J. Physiol. (Lond.) 387: 371-383.

Probert, L., J. DeMay, and J. M. Polak (1983) Ultrastructural localization of four different neuropeptides within separate populations of p-type nerves in the guinea pig colon. Gastroenterology 85: 10941104.

Role, L. W., and G. D. Fischbach (1987) Changes in the number of chick ciliary ganglion neuron processes with time in cell culture. $\mathrm{J}$. Cell. Biol. 104: 363-370.

Sah, D. W. Y., and S. G. Matsumoto (1987) Evidence for serotonin synthesis, uptake and release in dissociated rat sympathetic neurons in culture. J. Neurosci. 7: 391-399.

Said, S., and V. Mutt, eds. (1988) Vasoactive intestinal peptide and related peptides. Ann. NY. Acad. Sci. 527: 1-691.

Siegelbaum, S., J. Camardo, and E. Kandel (1982) Serotonin and cyclic AMP close single $\mathrm{K}^{+}$channels in Aplysia sensory neurones. Nature 299: 413-417.

Stanfield, P., Y. Nakajima, and Y. Nakajima (1985) Substance P raises neuronal excitability by reducing inward rectification. Nature 315 : 498-501.

Stollberg, J., P. Whiting, J. Lindstrom, and D. K. Berg (1986) Functional blockade of neuronal $\mathrm{ACh}$ receptors by antisera to a putative receptor from brain. Brain Res. 378: 179-182.

Walsh, J. P., and J. H. Byrne (1989) Modulation of a steady-state $\mathrm{Ca}^{2+}$-activated $\mathrm{K}^{+}$current in tail sensory neurons of Aplysia: Role of serotonin and cAMP. J. Neurophysiol. 61:32-44.

Willard, A. L., and R. Nishi (1985a) Neurons dissociated from rat myenteric plexus retain differentiated properties when grown in cell culture. II. Electrophysiological properties and responses to neurotransmitter candidates. Neuroscience 16: 201-211.

Willard, A. L., and R. Nishi (1985b) Neurons dissociated from rat myenteric plexus retain differentiated properties when grown in cell culture. III. Synaptic interactions and modulation by neurotransmitter candidates. Neuroscience 16: 213-221.

Willard, A. L., and R. Nishi (1987) Neuropeptides mark functionally distinguishable cholinergic enteric neurons. Brain Res. 422: 163-167.

Williams, J. T., and R. A. North (1979) VIP excites neurones of the myenteric plexus. Brain Res. 175: 174-177.

Yau, W. M., M. L. Youther, and P. R. Verdun (1985) A presynaptic site of action of substance $P$ and vasoactive intestinal polypeptide on myenteric neurons. Brain Res. 330: 382-385.

Zafirov, D. H., J. M. Palmer, P. R. Nemeth, and J. D. Wood. (1985) Bombesin, gastrin releasing peptide and VIP excite myenteric neurons. Eur. J. Pharmacol. 115: 103-107. 